\title{
Artigo Original / Original Paper \\ Germinación de Baccharis dracunculifolia subsp. tandilensis: caracterización basada en la temperatura, la luz y la salinidad
}

\author{
Germination of Baccharis dracunculifolia subsp tandilensis: characterization based \\ on temperature, light, and salinity
}

Vilma Teresa Manfreda ${ }^{1,3}$, María Luciana Alcaraz ${ }^{1}$ \& Rosa Lourdes Scaramuzzino ${ }^{1,2}$

\begin{abstract}
Baccharis dracunculifolia is a South American dioecious shrub of the Asteraceae family, with a wide geographic distribution and potential use for recovery of degraded areas, ornamental and medicinal purposes. There are two subspecies: $B$. dracunculifolia subsp. dracunculifolia and $B$. dracunculifolia subsp. tandilensis. The latter is endemic to the Tandilia hills system in Argentina and recently was recorded its expansion to another environment, the coastal dunes in the Buenos Aires province. The aims of this research were to characterize the germination of $B$. dracunculifolia subsp. tandilensis according to temperature and light, and to compare the germination in saline conditions of seeds coming from hills (A) and marine coastal dune environment (C). Germination responds positively to light without showing another important type of primary dormancy. Thermal time was $2500^{\circ} \mathrm{Ch}\left(104^{\circ} \mathrm{Cd}\right)$ and base temperature of $6^{\circ} \mathrm{C}$, without temperature germination constraints between $20-28^{\circ} \mathrm{C}$ and thermo-inhibition at $35^{\circ} \mathrm{C}$. Response to $\mathrm{NaCl}$ concentrations was similar between seeds from environments $\mathrm{A}$ and $\mathrm{C}$, both showed strong germination inhibition at $100 \mathrm{mM}$ of NaCl and higher concentrations. These results are discussed in relation to environmental conditions and in comparation whit physiological characteristics reported for subsp. dracunculifolia and other species within the genus Baccharis.
\end{abstract}

Key words: germination, thermal time, light-dark, salinity, Baccharis sp.

\section{Resumen}

Baccharis dracunculifolia es un arbusto dioico sudamericano de la familia Asteraceae, de amplia distribución geográfica con potencial uso en la recuperación de áreas degradadas, ornamental y medicinal. Existen dos subespecies: B. dracunculifolia subesp. dracunculifolia y B. dracunculifolia subesp. tandilensis. Esta última es endémica del sistema serrano de Tandilia y fue recientemente registrada su expansión a dunas costeras de la provincia de Buenos Aires. Los objetivos del trabajo fueron caracterizar la germinación de $B$. dracunculifolia subesp. tandilensis según la temperatura y luz, y comparar la germinación en condiciones salinas de semillas provenientes del ámbito serrano (A) con las de la costa marítima (C). Se pudo comprobar que la germinación responde positivamente a la luz y sin presentar otro tipo de dormición primaria de importancia. El tiempo térmico estimado fue $2500^{\circ} \mathrm{Ch}\left(104^{\circ} \mathrm{Cdía}\right)$ y la temperatura base de $6^{\circ} \mathrm{C}$, respondiendo al rango de 20 a $28^{\circ} \mathrm{C}$ con una germinación óptima y encontrándose termoinhibidas a $35^{\circ} \mathrm{C}$. La respuesta a diferentes concentraciones de $\mathrm{NaCl}$ fue similar entre las semillas procedentes de $\mathrm{A}$ y $\mathrm{C}$ : concentraciones de $100 \mathrm{mM}$ de $\mathrm{NaCl}$ y superiores fueron fuertemente inhibitorias de la germinación. Se discuten estos resultados relacionándolos con distintas condiciones ambientales y comparándolos con características fisiológicas de la germinación publicadas para la subsp. dracunculifolia y otras especies del género Baccharis.

Palabras clave: germinación, tiempo térmico, luz-oscuridad, salinidad, Baccharis sp.

\footnotetext{
'Universidad Nacional del Centro de la Provincia de Buenos Aires, Facultad de Agronomía - Biología General, República de Italia 780, Azul, Buenos Aires 7300, Argentina

2 ORCID: <https://orcid.org/0000-0002-6878-9998>

${ }^{3}$ Autor para correspondencia: vmanfred@faa.unicen.edu.ar
} 


\section{Introducción}

Baccharis dracunculifolia DC es un arbusto dioico sudamericano perteneciente a la familia Asteraceae, de amplia distribución geográfica. Actualmente se reconocen dos subespecies (Giuliano \& Plos 2014): B. dracunculifolia subsp. dracunculifolia, propia del sur de Brasil, Bolivia, Paraguay, Uruguay y norte y centro de la Argentina -hasta la región del Delta del río Paraná en la provincia de Buenos Aires-, y $B$. dracunculifolia DC subsp. tandilensis (Speg.) Giuliano que es endémica del sistema de Tandilia y regiones adyacentes de la provincia de Buenos Aires. Se han estudiado para la subespecie típica usos potenciales y reales que abarcan propiedades medicinales (Teixeira Duarte et al. 2005; da Silva Filho et al. 2009), aceites esenciales y/o uso como aromática (Ferracini et al. 1996; Lorenzo \& Paz 2004; Marchesi 2004; Fabiane et al. 2008), fuente de propóleo (Park et al. 2004), regeneración de ambientes (Galíndez et al. 2013; Negreiros et al. 2014), combustible (Giuliano \& Plos 2014). Se ha registrado para $B$. dracunculifolia subsp. tandilensis su uso local en florería y en la confección de ramos, y más ampliamente como ornamental (Dimitri 1988; Alonso et al. 2009; Hurrell et al. 2017), combustible (Frangi 1975) y como productor de aceite esencial (Prado et al. 2003).

La presencia de Baccharis dracunculifolia subsp. dracunculifolia ha sido constatada tanto en áreas degradadas con baja fertilidad como en campos agrícolas muy fértiles (Negreiros et al. 2014), y en diferentes relieves como sierras, dunas, planicies de derrame, llanuras aluviales (Brusa \& Grela 2007) demostrando así una alta capacidad colonizadora. En cuanto a Baccharis dracunculifolia subsp. tandilensis, denominada $B$. tandilensis durante el siglo XX, fue descripta por primera vez por Carlos Spegazzini (1901) y se la consideró endémica de la provincia de Buenos Aires (Zuloaga et al. 1999) y restringida al Sistema serrano de Tandilia (Crisci et al. 2001). Su presencia se asoció con afloramientos rocosos, grietas interbloques y pajonales serranos (Frangi 1975). En los últimos años se ha constatado una expansión de este taxón hacia regiones de llanura (Giuliano \& Plos 2014). Han sido observados, con presencia cada vez más frecuente, ejemplares aislados o arbustales incipientes ocupando pequeñas áreas en ambientes de llanura hacia el norte y el sur del sistema de Tandilia, llegando hasta la costa atlántica, generalmente en corredores (en las proximidades de rutas, caminos vecinales, vías férreas) y en algunos casos en pastizales (Scaramuzzino et al. 2015). Sus preferencias de hábitat se han ampliado. Actualmente se ubica a diferentes alturas sobre el nivel del mar, desde los casi $500 \mathrm{msnm}$ originarios en la cumbre de cerros hasta los $8 \mathrm{msnm}$ en la costa, y en diferentes suelos de los órdenes Molisoles y Entisoles (Scaramuzzino et al. 2015).

En cuanto al conocimiento biológico, se han efectuado numerosos estudios sobre $B$. dracunculifolia subsp. dracunculifolia, en particular o junto con otras especies del género, tanto en la Argentina como en Brasil. Las publicaciones abarcan temáticas como germinación (Gomes \& Fernandes 2002), alelopatía (Soares Gusman et al. 2008), fenología (Espírito-Santo et al. 2003), insectos asociados (Espírito-Santo \& Fernandes 1998; Fagundes \& Fernandes 2011), anatomía de tallo y hoja (Budel et al. 2004), respuesta de supervivencia y crecimiento de plántulas a gradiente de nutrientes (Negreiros et al. 2014), respuesta al fuego (Biganzoli et al. 2009; Galíndez et al. 2009; Galíndez et al. 2013), entre otras. Por el contrario, son escasas las investigaciones sobre $B$. dracunculifolia subsp. tandilensis y los existentes se han referido a aspectos taxonómicos (Spegazzini 1901; Cabrera 1963; Giuliano \& Plos 2014) o fitogeográficos-ecológicos (Cabrera 1958, 1976; Crisci et al. 2001; Scaramuzzino et al. 2015; Valicenti et al. 2005, 2010) estando ausentes los fisiológicos. Estos últimos ayudarían a comprender su actual expansión, siendo de particular interés las condiciones requeridas para la germinación y establecimiento de plántulas ya que se trata de una especie de diseminación por semilla. En casos como éste, cuando el material aún no ha sido caracterizado en estos aspectos, los criterios empleados en pruebas estandarizadas para especies relacionadas del mismo género o familia (como las del International Seed Testing Association -ISTA- para la valoración de calidad de semillas, de germinación y otras), resultan una importante herramienta, como lo destaca Araoz et al. 2016.

Entre los factores ambientales más importantes en determinar la tasa y el porcentaje de germinación se encuentran la temperatura y la disponibilidad de agua (Alvarado \& Bradford 2002). Según Dürr et al. (2015) la respuesta de la germinación a estos factores puede resumirse con la temperatura base, la suma térmica, las temperaturas cardinales y el potencial hídrico base, contribuyendo este conocimiento tanto a la comparación de especies 
como a la predicción de su distribución espacial. Sin embargo, estos parámetros sólo pueden ser evaluados cuando las semillas no presentan dormición (Batlla \& Benech-Arnold 2015). Entre los numerosos tipos de dormición (Baskin \& Baskin 2004; Finch- Savage \& Leubner-Metzger 2006) se encuentra la que responde a la luz, ampliamente reconocida como una señal de ambiente favorable a la germinación para especies cuyas semillas poseen un bajo nivel de reservas, como es el caso de $B$. medullosa y B. dracunculifolia (Galíndez et al. 2009).

A los requerimientos específicos para la germinación se suman las restricciones ambientales particulares, como la impuesta por suelos salinos. Las sales actúan bajando el potencial hídrico (Bradford 1995) disminuyendo y enlenteciendo la imbibición, mientras que, dependiendo de su composición iónica, pueden tener además efectos tóxicos (Begum et al. 2013). Entre los cationes el sodio es uno de los que más problemas induce (Azcón-Bieto \& Talón 2000) y, acompañado con el cloruro contribuyen mayormente a las sales de los ambientes salinos costeros. La tolerancia a la salinidad exhibe una considerable variabilidad entre especies (Glenn et al. 1999) y particularmente en el género Baccharis han sido constatadas varias especies como tolerantes tanto a la sequía como a la salinidad (ver Tucat 2015 y sus referencias; Cantero et al. 1990; Karrfalt \& Olson 2008).

Siendo las semillas la vía de propagación de esta especie y la germinación un período crítico para el establecimiento de las plantas, el conocimiento de los requerimientos para estas etapas puede explicar tanto la expansión en la distribución actual como sustentar la diferenciación entre ambas subespecies. Los objetivos de este trabajo están centrados en conocer la respuesta de la germinación a la temperatura y a la salinidad de $B$. dracunculifolia subsp. tandilensis, para la cual no hay datos, con el propósito de aportar desde su fisiología a la diferenciación de las dos subespecies y contribuir al entendimiento del aumento actual en su distribución territorial y hábitat.

\section{Materiales y Métodos}

\section{Aspectos generales}

Se plantearon dos ensayos diferentes con los aquenios dispuestos en cajas de Petri dentro de baños termostáticos y de cámaras de germinación, en uno de ellos con diferentes condiciones de temperatura y luz (Prueba de Germinación $=p G$ ), $\mathrm{y}$ en el otro con diferentes niveles de salinidad (Prueba de salinidad $=p S$ ).

\section{Material y condiciones de cultivo}

Se recolectaron ramas con aquenios de 6 plantas femeninas a principios del mes de noviembre de 2015 y de 2016 en las sierras de Azul (A) $\left(37^{\circ} 03^{\prime} \mathrm{S}, 59^{\circ} 45^{\prime} \mathrm{O}\right)$; y en octubre de 2016 sobre dunas de la costa de la provincia de Buenos Aires (C) (36 $\left.50^{\circ} \mathrm{S}, 5^{\circ} 42^{\prime} \mathrm{O}\right)$. Los ejemplares habían sido identificados y depositados en el herbario FAA (Thiers, continuamente actualizado) según los registros 7758 y 8599 . Los dos sitios presentan diferente tipo de suelo y altura sobre el nivel del mar. En las sierras los arbustos se disponen sobre suelos del orden Molisoles, en suelos con rocosidad y pedregosidad y en grietas, en alturas mayores a $200 \mathrm{msnm}$. En las dunas se ubican sobre suelos del orden Entisoles, altura a partir $11 \mathrm{msnm}$ y pendiente $0,5^{\circ}$ (Scaramuzzino et al. 2015). Los aquenios recolectados en 2015 utilizados para la $p G$, fueron almacenados durante 10 meses en bolsas de papel a temperatura ambiente antes de su uso. En cambio, los recolectados en 2016 fueron utilizados pocos días después de su recolección para la $p S$.

Las experiencias se condujeron con cajas de Petri de plástico de $9 \mathrm{~cm}$ de diámetro, con triple papel de filtro embebido en $3 \mathrm{ml}$ de agua desmineralizada (en $p G$ y $p S$ ) o con solución salina (en $p S$ ). En cada caja se sembraron 82 aquenios distribuyéndolos en forma regular en la superficie, seleccionados según las especificaciones generales para determinación de pureza en Asteraceae, ISTA (2010). A los fines de mantener constante la temperatura y humedad, las cajas de Petri se colocaron individualmente dentro de envases transparentes con tapa y sobre gomaespuma embebida en agua desmineralizada. Estos envases fueron colocados a su vez, en bandejas con agua (baños termostáticos) a diferentes temperaturas. Las temperaturas de los baños termostáticos fueron monitoreadas tanto con termómetro de mercurio como con un registrador digital que integró la temperatura de períodos de una hora. Las oscilaciones de las temperaturas no superaron $\operatorname{los} 2^{\circ} \mathrm{C}$ alrededor del valor nominal. La aplicación de luz blanca se realizó durante 16 horas diarias. La siembra de los tratamientos de oscuridad se realizó con luz verde, colocando las cajas de Petri en envases pintados de negro y cubriendo los baños termostáticos con plástico negro. 
Tratamientos y mediciones

Pruebas Germinación $(p G)$ : Las temperaturas (Temp) utilizadas fueron de: $7 ; 13 ; 20 ; 28$ y $35^{\circ} \mathrm{C}$, en combinación con dos tratamientos de iluminación (Ilum): suministro de luz desde la siembra (tratamiento L) o luego de un período de oscuridad (tratamiento OL). Cada tratamiento se realizó con 4 repeticiones asignando las cajas al azar. La duración del período oscuro se fijó a posteriori, cuando se consideró que el tratamiento L correspondiente a la misma temperatura finalizó su germinación (ver Tab. 1). A partir de ese recuento los aquenios de oscuridad comenzaron a recibir luz y se los siguió hasta que se consideró finalizada la germinación. Al concluir cada tratamiento se realizó una inspección para clasificar los aquenios según se detalla al final de este párrafo. La excepción a este diseño experimental fueron los tratamientos a $35^{\circ} \mathrm{C}(\mathrm{L}$ y OL) que se inspeccionaron a las 240 horas de la siembra ante una ausencia total de germinación. Se realizaron otras pruebas a los fines de confirmar los resultados del tratamiento $\mathrm{OL}$ a $7^{\circ} \mathrm{C}$ y ampliar los de $35^{\circ} \mathrm{C}$ (L y OL). En el primer caso se procedió de la forma antes descripta, agregando una envoltura de papel de aluminio a las cajas de Petri para reforzar el tratamiento de oscuridad. En el segundo caso, luego de permanecer por 840 horas
(36 días) a $35^{\circ} \mathrm{C}$, las muestras fueron transferidas a $20^{\circ} \mathrm{C}$ y luz permaneciendo bajo esta condición por una semana el tratamiento L y por dos el OL. Transcurridos esos tiempos se procedió a clasificar el estado de los aquenios en cuatro categorías: con semilla germinada $(\mathrm{G})$, con semilla con hongos o putrefacta (M), con semilla firme (F) o como fruto vano o vacío $(\mathrm{V})$. Se consideró como criterio de germinación o semilla germinada aquella con la radícula emergiendo a través de los tegumentos. La disección bajo microscopio estereoscópico $(20 \times)$ diferenció a los aquenios $\mathrm{V}$ por su falta de contenido. Las semillas F, consideradas como vivas, presentaron coloración blanquecina y resistencia a la presión. En contraposición, las semillas $\mathrm{M}$ (muertas no viables) se caracterizaron por ser blandas y deshacerse fácilmente bajo presión, acompañada esta característica con una coloración oscura y/o con presencia de hongos.

Pruebas de Salinidad $(p S)$ : Se sembraron en las cajas de Petri por separado aquenios colectados en las sierras (A) y en la costa (C), en las cuales se utilizaron soluciones de $0 ; 50 ; 100 ; 150$ y 200 $\mathrm{mM}$ de $\mathrm{NaCl}$. Cada condición de origen de los aquenios y concentración salina fue analizada por cuadruplicado. La temperatura empleada fue de $20^{\circ} \mathrm{C}$ y se proveyó de luz blanca por 16 horas

Tabla 1 - Porcentaje de germinación promedio al final del período de oscuridad (\%Gosc) y del de luz $(\% \mathrm{G})$ con la duración bajo cada condición lumínica expresado en horas (O-L) para las diferentes temperaturas. Los promedios de porcentajes de semillas muertas $(\% \mathrm{M})$ y firmes $(\% \mathrm{~F})$ fueron obtenidos al finalizar los tratamientos de luz. Letras diferentes dentro e cada columna indican diferencias significativas obtenidas con la prueba de Scheffé $(\alpha \leq 0,05)$. Factores: Temp $=$ tratamiento de temperatura $\left({ }^{\circ} \mathrm{C}\right) ; \mathrm{Ilum}=$ Tratamientos de iluminación; $\mathrm{L}=1 \mathrm{uz} ; \mathrm{OL}=$ período de oscuridad contínua-período con fotoperíodo diario (en horas).

Table 1 - Mean percentage of germination obtained at the end of dark period (\% Gosc) and light period (\% G) and time elapsed under each lighting condition in hours (O-L) at different temperatures. Mean percentages of dead ( $\%$ M) and firm (\% F) seeds were evaluated at the end of lighting treatments. Different letters within each column represent significant differences obtained by Scheffé test ( $\alpha \leq 0.05$ ). Factors: Temp = temperature treatment; Ilum = lighting treatments; $\mathrm{L}=$ light; $\mathrm{OL}=$ continuous dark period-period with daily photoperiod.

\begin{tabular}{lllllll}
\hline Temp $\left({ }^{\circ} \mathbf{C}\right)$ & Ilum & O-L & \%Gosc & \%G & \%M & \%F \\
\hline 7 & L & $0-1466$ & ----- & $75,15 \mathrm{bc}$ & $12,29 \mathrm{ab}$ & $11,56 \mathrm{a}$ \\
7 & OL & $1012-1436$ & $22,24 \mathrm{a}$ & $61,26 \mathrm{~b}$ & $20,51 \mathrm{a}$ & $18,23 \mathrm{a}$ \\
13 & $\mathrm{~L}$ & $0-474$ & ----- & $78,55 \mathrm{bc}$ & $14,00 \mathrm{ab}$ & $7,45 \mathrm{a}$ \\
13 & OL & $410-474$ & $0,45 \mathrm{a}$ & $87,99 \mathrm{c}$ & $8,83 \mathrm{ab}$ & $3,18 \mathrm{a}$ \\
20 & $\mathrm{~L}$ & $0-188$ & ----- & $90,39 \mathrm{c}$ & $5,54 \mathrm{ab}$ & $4,07 \mathrm{a}$ \\
20 & OL & $216-188$ & $1,72 \mathrm{a}$ & $87,31 \mathrm{c}$ & $5,12 \mathrm{ab}$ & $7,58 \mathrm{a}$ \\
28 & $\mathrm{~L}$ & $0-188$ & ----- & $71,80 \mathrm{bc}$ & ----- & ----- \\
28 & OL & $360-188$ & $0,82 \mathrm{a}$ & $77,15 \mathrm{bc}$ & $7,13 \mathrm{ab}$ & $15,72 \mathrm{a}$ \\
35 & $\mathrm{~L}$ & $0-432$ & ------ & $0,00 \mathrm{a}$ & $17,59 \mathrm{ab}$ & $82,41 \mathrm{~b}$ \\
35 & OL & $432-0$ & $0,00 \mathrm{a}$ & $0,00 \mathrm{a}$ & $2,65 \mathrm{~b}$ & $97,35 \mathrm{~b}$ \\
\hline
\end{tabular}


diarias. A los 16 días (348 horas) desde la siembra se procedió a la inspección de las cajas clasificando los aquenios según las categorías utilizadas en la $p G$.

\section{Análisis de los datos}

La comparación del porcentaje de aquenios vanos de diferente procedencia u origen se realizó mediante la prueba de Student a dos colas. Los porcentajes del resto de categoría de aquenios se hicieron excluyendo a los vacíos y fueron analizados mediante un análisis de la varianza (ANOVA) según la temperatura y tratamiento de iluminación en la $p G$ y según origen y salinidad en la $p S$. Los contrastes fueron realizados con la prueba de Scheffé.

En la $p G$ los porcentajes de germinación $(\% \mathrm{G})$ se ajustaron a lo largo del tiempo (T) mediante un modelo no lineal. Para este análisis se sustrajeron del cálculo los aquenios vacíos y para los tratamientos OL también las semillas germinadas durante el período de oscuridad. Para los tratamientos OL el tiempo utilizado fue contabilizado a partir de iniciarse la iluminación. Cada unidad experimental se ajustó a la siguiente ecuación:

$$
\% \mathrm{G}=\mathrm{A}^{*}\left(1-1 /(1-\mathrm{T} / \mathrm{Tm})^{\mathrm{k}}\right)
$$

En la que: A es el porcentaje de germinación máximo alcanzado (valor asintótico de la curva), Tm el Tiempo medio de Germinación (horas al $50 \%$ de germinación) y $\mathrm{k}$ es un coeficiente de velocidad de germinación (Manfreda, manuscrito en preparación). Los valores de parámetros obtenidos para cada muestra fueron analizados mediante ANOVA y comparados entre temperaturas e iluminación con el test de Scheffé.

Para los análisis antes citados y los de normalidad y homocedacia, se utilizó el paquete estadístico InfoStat versión 2016 (Di Rienzo et al. 2016). Cuando las variables se alejaron de los supuestos de normalidad y homocedacia se aplicaron las transformaciones adecuadas.

\section{Resultados y Discusión}

\section{Prueba Germinación $(p G)$}

a-1 Estado final de las semillas: La inspección final de los aquenios obtenidos en las sierras de Azul, mostró que el porcentaje de frutos vacíos promedió el $33 \%$, con muestras con sólo un $13 \%$ y otras que llegaron al $65 \%$. La alta variabilidad entre muestras llevó a corregir los datos de cada una de ellas según la cantidad particular de aquenios vanos. Valores tan altos y variables de semillas vacías han sido obtenidos en distintas especies de Baccharis (Tardáguila 2004; Aráoz et al. 2016) atribuyendo a este factor los bajos porcentajes de germinación obtenidos.

Los $\% \mathrm{G}$ alcanzados al finalizar el período de luz, no mostraron efectos del factor Iluminación, y por el contrario el efecto de la temperatura fue altamente significativo (resultados del ANOVA no mostrados, $\alpha \leq 0,05)$. En el rango entre 13 y $28^{\circ} \mathrm{C}$ los porcentajes de germinación fueron altos y no se detectaron efectos de la temperatura. Dentro de ese rango de temperaturas, e incluyendo a $7^{\circ} \mathrm{C}$, tanto la categoría de semillas firmes como muertas no variaron $(\alpha=0,05$, Tab. 1$)$. La presencia de semillas firmes al finalizar las pruebas indica la presencia de otro tipo de dormición o de bajo vigor, que estaría afectando a un promedio relativamente bajo de simientes (Tab. 1). A $35^{\circ} \mathrm{C}$ la germinación fue nula y diferente a la registrada en temperaturas medias y bajas (Tab. 1). Esta falta de germinación se correspondió con un alto porcentaje de semillas firmes $(\alpha=0,05$, Tab. 1$)$. En el tratamiento OL a $7^{\circ} \mathrm{C}$ se observó un $\% \mathrm{G}$ relativamente bajo, pero en este caso se correspondió tanto con el $\% \mathrm{M}$ como con el \%F (Tab. 1).

Difiriendo de estas respuestas a la temperatura, Gomes \& Fernandes (2002) en un rango entre 15 y $30^{\circ} \mathrm{C}$ y en presencia de luz, obtuvieron para aquenios de Baccharis dracunculifolia recientemente cosechados, un mayor porcentaje de germinación a 15 y a $20^{\circ} \mathrm{C}$. Estas observaciones las realizaron luego de un período de tiempo similar o superior al aquí empleado (30 días - 720 horas) y los valores de germinación fueron altos, por lo que se supone que corresponden a una germinación finalizada plenamente y en ausencia de dormición, al menos en el rango de temperaturas bajas. Si bien estos elementos habilitan a realizar comparaciones con los resultados de este trabajo, esta comparación queda limitada por la falta de evaluación del estado de los aquenios de los autores, por lo que no es posible atribuir las diferencias solamente a un único factor. Bajo estas consideraciones sólo podemos afirmar que en el presente trabajo encontramos que el rango de temperaturas con alto porcentaje de germinación fue más amplio que el de Gomes \& Fernandes (2002) y que la germinación fue nula a la mayor temperatura, este último punto se discute más adelante.

La importancia de la luz como requerimiento para la germinación de $B$. dracunculifolia subsp. tandilensis, queda expuesta al comparar los \%G obtenidos al finalizar el período de oscuridad 
y luego de aplicar luz a las mismas unidades experimentales (Tab. 1, columna \%Gosc y $\% \mathrm{G})$. La germinación registrada al finalizar el período de oscuridad (\%Gosc), fue nula o muy baja y los porcentajes no mostraron diferencias significativas entre temperaturas $(\alpha=0,05$; Tab. 1). Cabe recordar que la duración del período de oscuridad fue fijada para cada temperatura al momento en que se completó la germinación del correspondiente tratamiento $\mathrm{L}$. La dependencia a la luz es frecuente encontrarla en especies que poseen semillas de muy baja masa (JankowskaBlaszczuk \& Daws 2007; Galíndez et al. 2009; Koutsovoulou et al. 2014) y es característica del género Baccharis (Karrfalt \& Olson 2008). Esta respuesta positiva de la germinación a la iluminación fue encontrada en aquenios de $B$. dracunculifolia subsp. dracunculifolia recolectados en la provincia de Entre Ríos (31 ${ }^{\circ} 55^{\prime} \mathrm{S}$; 58 $8^{\circ} 17^{\prime} \mathrm{O}$, Galíndez et al. 2009) tanto con un régimen térmico alternante $\left(8\right.$ horas a $25^{\circ} \mathrm{C}$ y 16 horas a $\left.10^{\circ} \mathrm{C}\right)$ como bajo uno constante a $20^{\circ} \mathrm{C}$. Sin embargo, Gomes $\&$ Fernandes (2002) encontraron que, para la misma subespecie, a $15^{\circ} \mathrm{C}$ la germinación ocurría independientemente de la luz con muy altos valores porcentuales, mientras que a $20 ; 25$ y $30^{\circ} \mathrm{C}$ fue significativamente mayor en luz que en oscuridad, por lo que concluyeron sobre la existencia de una interacción entre la temperatura y la luz. Nuestros resultados a $13^{\circ} \mathrm{C}$ y, con menor certidumbre a $7^{\circ} \mathrm{C}$, no muestran esa interacción. Si bien la germinación en el tratamiento a $7^{\circ} \mathrm{C}$ en oscuridad resultó en un porcentaje promedio relativamente alto, esto se presentó junto a una alta dispersión entre muestras $(0 ; 6,88 ; 13,85$ y $69,2 \%)$, lo que llevó a suponer una falla en la aplicación de la oscuridad en dos de las repeticiones, poniendo en dudas la interacción entre la temperatura y el requerimiento de luz. La confirmación a tal suposición se obtuvo con la repetición del tratamiento de $\mathrm{OL}$ a $7^{\circ} \mathrm{C}$ que resultó en una germinación nula al finalizar el período de oscuridad y con un $\% \mathrm{~V}$ de 8,44 y desv std de 3.62 . Las discrepancias referentes a la interacción luztemperatura entre los aquenios aquí analizados y los informados para la subespecie dracunculifolia podrían obedecer a diferencias, ya sea en relación a las subespecies y/o a las condiciones durante la formación de las semillas.

Con respecto a los resultados del extremo superior del rango de temperaturas, podría esperarse que la alta temperatura, en conjunto con alta humedad, principios en los que se basan pruebas de envejecimiento de semillas, de longevidad, etc., favorezcan el deterioro y posterior muerte seminal (TeKrony 2005), en particular con semillas de baja permanencia en los bancos de semillas, como es el caso de las de B. dracunculifolia (Galíndez 2008; Galíndez et al. 2013). Este último hecho no se presentó ni en la temperatura más alta utilizada (Tab. 1). Probablemente sean necesarias condiciones más extremas y/o sostenidas por más tiempo que las empleadas en este trabajo cuyas temperaturas son más acordes con las de primaveraverano, estación a las que están expuestas las semillas recién producidas. Dado el alto porcentaje de semillas vivas pero no germinadas encontrado a $35^{\circ} \mathrm{C}$ (tanto en L como en OL, Tab. 1), se realizó una prueba adicional a fin de definir el estado entre quiescente o dormición.

La exposición a $35^{\circ} \mathrm{C}$ por 36 días de las semillas (con ausencia absoluta de germinación) y su pasaje a $20^{\circ} \mathrm{C}$ y luz por una semana, llevó al tratamiento L a una germinación del $66 \%$, quedando como remanente un 15 y $18 \%$ de F y $\mathrm{M}$ respectivamente. El cambio en la temperatura y la exposición a la luz del tratamiento OL condujo, luego de dos semanas, a valores superiores de germinación a los obtenidos en el tratamiento L $(93,5 \% ; \alpha \leq 0.05)$ acompañados de un $3,8 \%$ de F y $2,7 \%$ de M. Tanto para L como para OL los resultados de los \% de F y M se encuentran dentro del rango de valores encontrados entre 13 y $28{ }^{\circ} \mathrm{C}$ (ver Tab. 1). Este efecto inhibitorio de la germinación ejercido por la alta temperatura durante la imbibición, y que es rápidamente revertido por exposición a temperaturas menores es llamado termohinhibición. Fue registrado en un amplio conjunto de especies que incluyen especies cultivadas, como lechuga y zanahoria, como a otras anuales invernales de climas mediterráneos (Huo \& Bradford 2015). En las cultivadas esta inhibición es indeseada ya que va en detrimento del establecimiento del cultivo cuando éste se siembra a temperaturas altas. En cambio, en climas mediterráneos, la inhibición actuaría impidiendo la germinación en los veranos secos y la favorecería en el otoño cuando las temperaturas disminuyen y la probabilidad de lluvias es mayor. Esta situación es la descripta por Gomes \& Fernandes (2002) para la zona de extracción de los aquenios de su trabajo. En la provincia de Buenos Aires, si bien presenta una distribución de lluvias con precipitaciones abundantes desde la primavera temprana hasta fines del verano-principios de otoño (marzo-abril), en los meses de diciembre, enero y febrero el déficit hídrico supera el 20\% (Sala et al. 1987; Requesens 
2011), restringiendo fuertemente la disponibilidad de agua en el suelo. Es entonces probable que el ajuste propuesto opere también en este caso.

Los resultados muestran al requerimiento de luz como la principal causa de dormición primaria, por lo cual con la velocidad de germinación medida bajo iluminación es posible estimar las temperaturas cardinales de esta etapa del desarrollo y el tiempo térmico, pudiéndose anticipar que la temperatura máxima se encuentra dentro del rango de 28 a $35^{\circ} \mathrm{C}$. Ya que, $35^{\circ} \mathrm{C}$ es una temperatura que excede la temperatura máxima de la germinación, y por tanto cae por fuera de la linealidad del modelo a utilizar para estimar la temperatura base (Tb) y la suma térmica (TT), los resultados obtenidos en esta temperatura se los excluyen de los siguientes análisis.

a-2 Curvas de germinación en el tiempo: La curva usada para el seguimiento en el tiempo del \%G satisfizo los siguientes criterios: 1) presentó un menor cuadrado medio del error que la obtenida mediante la función de Gompertz, función recomendada por Balzarini et al. (2008) para el seguimiento de la germinación en el tiempo (datos no mostrados); 2) a igualdad de cantidad de parámetros a ser estimados, las constantes de esta ecuación tienen un significado biológico fácilmente identificable; y 3) en todos los casos se comprobó que el seguimiento abarcó el acercamiento asintótico al \%Gfinal.

Los $\% \mathrm{G}$ final obtenidos para los tratamientos L y OL mediante el ajuste a la curva (Alfa), mostraron un efecto significativo de interacción entre la temperatura y la iluminación $(\alpha=0,05$, Tab. 2). Los contrastes mostraron diferencias significativas solamente entre el tratamiento $\mathrm{L}$ a $7{ }^{\circ} \mathrm{C}$ con el de $\mathrm{OL}$ a $13{ }^{\circ} \mathrm{C}$, con el menor y mayor valor respectivamente $(\alpha=0,05$, Tab. 3 ). Estas diferencias no fueron detectadas en la comparación de promedios antes presentadas debido, probablemente, a la inclusión en ese caso de los datos de $35^{\circ} \mathrm{C}$.

El coeficiente de velocidad $(K)$ está relacionado con la dispersión en el tiempo de la germinación: cuanto más breve es el período entre el inicio y el final de la germinación, mayor es el valor del coeficiente. La comparación de este parámetro mostró que es afectado únicamente por la iluminación mientras que la temperatura no tuvo por sí misma, ni en interacción con la iluminación efectos sobre el mismo (Tab. 2). Si bien el contraste agrupó a todos los tratamientos L, sólo diferenció claramente a $\mathrm{OL}$ obtenido a $7{ }^{\circ} \mathrm{C}$ con un menor valor de $\mathrm{K}$, debiéndose entonces considerarlo con la misma cautela que en el caso anterior (Tab. 3). Aun así, se puede observar una tendencia a germinar en forma más dispersa en el tiempo a las semillas bajo el tratamiento OL que las que recibieron luz desde el inicio de la hidratación (Tab. 3).

Los tiempos medios determinados por el ajuste a la función y empleando el tiempo transcurrido desde el inicio de la iluminación, se alejaron de los supuestos de normalidad y homocedacia requeridos para la comparación estadística $(\alpha=0,05)$. Esta variable se normalizó mediante el cálculo de su inversa, transformación que no afecta su interpretación biológica ya que es una medida de la velocidad de desarrollo, en este caso la velocidad al $50 \%$ de la germinación (V50\%). Se encontraron efectos significativos

Tabla 2 - Análisis de la varianza de los parámetros de las curvas del porcentaje de germinación. Alfa es el valor asintótico correspondiente al porcentaje final de germinación. V50\% es la velocidad de germinación estimada cuando el 50\% de las semillas han germinado (inversa del Tmed de la curva). K es el coeficiente de curvatura de la función. Table 2 - Analysis of variance for parameters estimated by adjusted percentage of seed germination to time curve. Alpha is the asymptotic value corresponding to the final percentage of germination. V50\% is the germination rate at $50 \%$ of seeds germinated (inverse of Tmed of the curve). $\mathrm{K}$ is the coefficient of curvature of function.

\begin{tabular}{llllclll}
\hline F.V. & \multicolumn{3}{c}{ Alfa } & \multicolumn{2}{c}{ V50\% } & \multicolumn{2}{c}{ K } \\
\hline & gl & F & p-valor & F & p-valor & F & p-valor \\
Modelo & 7 & 3,24 & 0,0120 & 299,87 & $<0,0001$ & 7,19 & 0,0001 \\
Temp & 3 & 4,13 & 0,152 & 621,77 & $<0,0001$ & 1,41 & 0,2600 \\
Ilum & 1 & 1,96 & 0,1728 & 214,42 & $<0,0001$ & 42,31 & $<0,0001$ \\
Temp*Ilum & 3 & 3,11 & 0,0422 & 42,84 & $<0,0001$ & 1,18 & 0,3350 \\
Error & 28 & & & & & & \\
Total & 35 & & & & & & \\
\hline
\end{tabular}


Tabla 3 - Promedios de las constantes de la función que describe los cambios del $\% \mathrm{G}$ a lo largo del tiempo y los contrastes realizados con la prueba de Scheffé $(\alpha=0,05)$. Los valores de Alfa corresponden al \%G final alcanzado, V50\% es la inversa del período de tiempo empleado para llegar al 50\% del Alfa y K es un coeficiente de curvatura. Letras diferentes dentro de cada columna indican diferencias significativas obtenidas con la prueba de Scheffé $(\alpha \leq 0,05)$. Tratamientos: Temp $=$ temperatura $\left({ }^{\circ} \mathrm{C}\right)$; Ilum= iluminación; $\mathrm{L}=1 \mathrm{luz} ; \mathrm{OL}=$ período de oscuridad total-período con fotoperíodo diario (en horas).

Table 3 - Mean of value parameters of the function that describes the $\%$ G over time and the contrasts performed with the Scheffé test $(\alpha=0.05)$. The alpha values correspond to the final $\% \mathrm{G}$ achieved, V50\% is the inverse of the time period used to reach $50 \%$ of the alpha and $\mathrm{K}$ is a coefficient of curvature. Different letters within each column indicate significant differences obtained with the Scheffé test $(\alpha \leq 0.05)$. Factors: Temp $=$ temperature; Ilum $=$ lighting; $\mathrm{L}=$ light; $\mathrm{OL}=$ total dark period-period with daily photoperiod.

\begin{tabular}{lllll}
\hline Temp $\left({ }^{\circ} \mathbf{C}\right)$ & Ilum & Alfa & V50\% & K \\
\hline 7 & L & $67,79 \mathrm{a}$ & $1,031 \mathrm{a}$ & $10,11 \mathrm{~b}$ \\
7 & OL & $81,51 \mathrm{ab}$ & $1,651 \mathrm{ab}$ & $3,98 \mathrm{a}$ \\
13 & $\mathrm{~L}$ & $80,91 \mathrm{ab}$ & $3,126 \mathrm{bc}$ & $10,40 \mathrm{~b}$ \\
13 & OL & $96,91 \mathrm{~b}$ & $4,071 \mathrm{c}$ & $6,73 \mathrm{ab}$ \\
20 & $\mathrm{~L}$ & $83,64 \mathrm{ab}$ & $7,944 \mathrm{~d}$ & $10,86 \mathrm{~b}$ \\
20 & OL & $76,88 \mathrm{ab}$ & $14,665 \mathrm{f}$ & $6,46 \mathrm{ab}$ \\
28 & $\mathrm{~L}$ & $76,45 \mathrm{ab}$ & $10,467 \mathrm{e}$ & $10,08 \mathrm{~b}$ \\
28 & OL & $72,20 \mathrm{ab}$ & $14,861 \mathrm{f}$ & $7,24 \mathrm{ab}$ \\
\hline
\end{tabular}

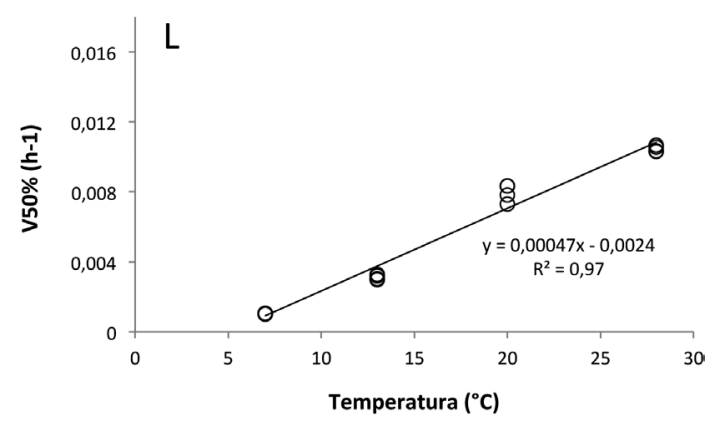

de las temperaturas, el tratamiento de luz y de su interacción (Tab. 2). Los valores más altos se obtuvieron luego de la exposición a la luz en los tratamientos OL en las dos temperaturas más altas $\left(20\right.$ y $\left.28^{\circ} \mathrm{C}\right)$. A éstos los siguieron los $\mathrm{L}$ correspondientes a esas dos temperaturas, y finalmente los de $13 \mathrm{y}$ de $7{ }^{\circ} \mathrm{C}$ sin diferir dentro de cada temperatura entre los tratamientos de iluminación (Tab. 3).

a-3 Estimación de la Temperatura base y Suma Térmica de la germinación: El gráfico de la V50\% con la temperatura permitió observar que

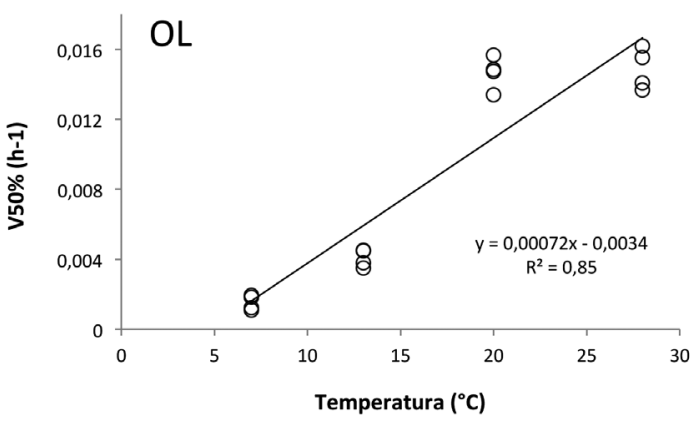

Figura 1 - Relación entre la velocidad de germinación (V50\%) y la temperatura para los tratamientos lumínicos: luz (L) y oscuridad seguida por luz (OL).

Figure 1 - Relationship between germination rate (V50\%) and temperature for lighting treatments: light (L) and dark followed by light $(\mathrm{OL})$. los datos correspondientes a $28{ }^{\circ} \mathrm{C}$ pertenecían a la recta de respuesta positiva a la temperatura (Fig. 1a). La regresión lineal obtenida fue altamente significativa $(\alpha<0.0001)$. Se siguió el mismo criterio para analizar los datos obtenidos en el tratamiento OL, para el que también la regresión fue significativa $(\alpha<0.0001$, Fig. 1b). La temperatura base estimada a partir de estas regresiones fue de $6{ }^{\circ} \mathrm{C}$ y la suma térmica para la germinación fue de $2500{ }^{\circ} \mathrm{C} . \mathrm{h}\left(104{ }^{\circ} \mathrm{Cd}\right)$ cuando las semillas fueron provistas con luz desde la imbibición. Para los tratamientos OL la temperatura base estimada fue de $4,7^{\circ} \mathrm{C}$ y el TT para la germinación de $1388^{\circ} \mathrm{C} . \mathrm{h}$ (aproximadamente $58^{\circ} \mathrm{C} . \mathrm{d}$ ) estimaciones realizadas considerando el tiempo a partir del momento en que se les suministró luz. El acortamiento del TT 
indica que parte de los eventos que conducen a la germinación fueron cumplimentados durante el período de oscuridad y presumiblemente con una temperatura base mayor a $4,7{ }^{\circ} \mathrm{C}$, lo que llevó a calcular para los tratamientos $\mathrm{L}$ una temperatura base mayor.

Según estos resultados B. dracunculifolia subsp. tandilensis se clasificaría como una especie de germinación intermedia, según los tiempos de germinación, (Ferreira et al. 2001) y según los TTmedio (Dürr et al. 2015). Esto implica que un alto porcentaje de germinación se obtendría en una ventana de tiempo estrecha con condiciones ambientales favorables, tales como las que se requieren en ambientes o micrositios con disponibilidad de agua muy fluctuante y dependiente de la lluvia.

\section{Prueba Salinidad ( $p S)$}

La prueba de salinidad, realizada a $20^{\circ} \mathrm{C}$ con aquenios cosechados poco tiempo antes de realizar la prueba, mostró que el porcentaje de aquenios vanos (V) fue diferente según la localidad de extracción $(\alpha \leq 0,0001)$. Para A el valor promedio fue de sólo un 18\%, mientras que para los extraídos en C llegó a 49\%. Tanto la diferencia en los valores promedio como la alta variabilidad interna, confirmaron la importancia de basar los cálculos del estado de las semillas sobre muestras grandes de aquenios y de hacer las correcciones pertinentes para cada muestra en particular, correcciones que se hicieron para los resultados que se exponen a continuación.

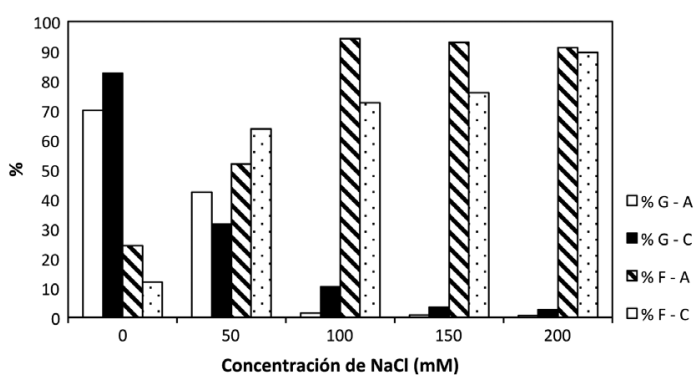

Figura 2 - Porcentajes de semillas germinadas $(\% \mathrm{G})$ y firmes $(\% \mathrm{~F})$ obtenidas de aquenios cosechados en las sierras de Azul (A) y en las dunas de la costa atlántica $(\mathrm{C})$ luego de la exposición a diferentes soluciones de cloruro de sodio $(\mathrm{NaCl})$.

Figure 2 - Percentages of germinated $(\% \mathrm{G})$ and firm $(\% \mathrm{~F})$ seeds obtained from achenes harvested in Sierras de Azul (A) and in dunes of the Atlantic coast (C) after exposure to different solutions of sodium chloride $(\mathrm{NaCl})$.
La exposición al $\mathrm{NaCl}$ causó un efecto significativo sobre el $\% \mathrm{G}$, mientras que el origen de la simiente y la interacción entre estos dos factores no lo modificaron (Tab. 4). Los promedios de $\% \mathrm{G}$ en $0 \mathrm{mM}$ (testigos) fueron de $86 \%$ y $69 \%$ para $\mathrm{C}$ y $\mathrm{A}$ respectivamente, no alcanzando la diferencia entre los valores a ser significativa $(\alpha=0,05)$. Con el aumento de la salinidad los $\% \mathrm{G}$ disminuyeron llegando a ser prácticamente nulos en 150 y $200 \mathrm{mM}$ de $\mathrm{NaCl}$ (Fig. 2). En esta prueba la imposición de la salinidad no aumentó el porcentaje de semillas muertas, tampoco se encontraron para el mismo diferencias según la localidad de origen de las simientes $(6,0 \%$ y $13,8 \%$ para A y $\mathrm{C}$ respectivamente; Tab. 4). En contraposición, el porcentaje de semillas firmes fue modificado muy significativamente por la salinidad (Tab. 4) y en correspondencia con la disminución de las semillas germinadas, tanto para las de origen en las dunas costeras como las de las sierras (Fig. 2).

Estos resultados son compatibles con la hipótesis que sostiene que en ambientes más estresantes, como el salino de la costa, la producción de semillas viables es menor (Munns \& Rawson 1999). Esta especie ha sido reconocida por su amplia distribución y como colonizadora de muy variados ambientes que incluyen una amplia gama de diferentes disponibilidades de nutrientes minerales (Negreiros et al. 2014). Varias de las numerosas especies de este género han sido usadas para regenerar áreas disturbadas por su capacidad de adaptarse a condiciones adversas, incluidas algunas con capacidad de colonizar dunas bajo influencia marina (Karrfalt \& Olson 2008; Montalvo et al. 2010; Hester et al. 2012). La distribución conocida para $B$. dracunculifolia subsp. tandilensis hasta hace poco no evidenciaba esta capacidad para afrontar los desafíos que representan para la germinación, crecimiento y desarrollo las condiciones de las dunas costeras marinas. Espírito-Santo et al. (2003) han informado sobre el alto costo metabólico que supone la reproducción para los pies femeninos de B. dracunculifolia subsp. dracunculifolia. La alta asignación de recursos para hacerlo llega a ocasionar la muerte de ramas e incluso de individuos, en forma particular bajo estrés hídrico. Este resultado es bien conocido en especies cultivables en las que, previamente a la muerte de las plantas, la disminución de los recursos asignados para la reproducción, conlleva tanto a la disminución en la calidad como en la producción de semillas Este efecto de factores ambientales de estrés sobre la asignación de recursos a la reproducción puede hacerse extensivo a especies 
Tabla 4 - Análisis de la varianza de los porcentajes de germinación $(\% \mathrm{G})$, de semillas muertas o vanas $(\% \mathrm{M})$, y de semillas firmes $(\% \mathrm{~F})$, de aquenios según su ambiente de procedencia (Localidad= Loc) y concentraciones de $\mathrm{NaCl}$ en el medio de germinación (Sal).

Table 4 - Analysis of variance of germination ( $\% \mathrm{G})$, dead and empty seeds ( $\% \mathrm{M})$, and firm seeds $(\% \mathrm{~F})$ in percentages of achenes by their environmental (Localidad $=\mathrm{Loc}$ ) and $\mathrm{NaCl}$ concentrations in germination medium (Sal).

\begin{tabular}{|c|c|c|c|c|c|c|c|}
\hline \multirow[t]{2}{*}{ F.V. } & \multicolumn{3}{|c|}{$\% G$} & \multicolumn{2}{|c|}{$\% M$} & \multicolumn{2}{|c|}{$\% \mathrm{~F}$} \\
\hline & gl & $\mathbf{F}$ & p-valor & $\mathbf{F}$ & p-valor & $\mathbf{F}$ & p-valor \\
\hline Modelo & 9 & 18,57 & $<0,0001$ & 0,84 & 0,5827 & 9,53 & $<0,0001$ \\
\hline Origen & 1 & 0,44 & 0,5104 & 1,74 & 0,1972 & 1,84 & 0,1853 \\
\hline Sal & 1 & 40,90 & $<0,0001$ & 0,65 & 0,6283 & 19,99 & $<0,0001$ \\
\hline Origen*Sal. & 4 & 0,77 & 0,5508 & 0,81 & 0,5289 & 1,00 & 0,4210 \\
\hline Error & 30 & & & & & & \\
\hline Total & 39 & & & & & & \\
\hline
\end{tabular}

silvestres, tales como los observados en la asteracea Flourensia cernua (Valencia-Díaz \& Montaña 2005) y en la más emparentada $B$. ulicina (Tucat 2015). El aumento de frutos vacíos tal como ocurre en situación de estrés hídrico para los dos casos citados, bien podría explicar la mayor proporción de aquenios vacíos observada en la costa. Por otro lado, no se registraron diferencias en la capacidad de germinación bajo salinidad, que pudieran sugerir hipótesis respecto a una tolerancia o resistencia de origen genético o de efecto materno, en las semillas provenientes de las plantas de la costa con respecto a las de origen serrano.

Los resultados muestran que $B$. dracunculifolia subsp. tandilensis, tiene un mayor requerimiento de luz para germinar que el reportado para la subespecie típica, en la que este requerimiento presenta interacción con la temperatura. Además, no presenta otro tipo de dormición primaria de importancia y por sus temperaturas cardinales, en particular la temperatura base de $4,7{ }^{\circ} \mathrm{C}$ en oscuridad seguida por luz y de $6^{\circ} \mathrm{C}$ bajo luz, podría encuadrarse en las típicas para una especie templada. Sin embargo, la presencia de termoinhibición la ubica en ambientes con estrés simultáneo hídrico y alta temperatura, en concordancia con los ambientes de dunas costeras en que su aparición es de registro reciente. Por otro lado, no se han encontrado diferencias en la germinación en presencia de $\mathrm{NaCl}$ que pudieran indicar un mecanismo particular para afrontar este estrés en los aquenios obtenidos en la costa atlántica.

\section{Agradecimientos}

Para los revisores del trabajo cuyas observaciones contribuyeron a mejorarlo sustancialmente y al Editor que amable y solícitamente atendió nuestras consultas. Agradecemos también a la Universidad Nacional del Centro de la Provincia de Buenos Aires por sostener, con sus escasos recursos, actividades de investigación y, finalmente a este medio de difusión por su carácter abierto y gratuito.

\section{Referencias}

Alonso SI, Nuciari MC, Guma IR \& van Olphen A(2009) Flora de un área de la Sierra La Barrosa (Balcarce) y fenología de especies con potencial ornamental. Revista de la Facultad de Ciencias Agrarias XLI: 23-44.

Alvarado V \& Bradford KJ (2002) A hydrothermal time model explains the cardinal temperatures for seed germination. Plant, Cell \& Environment 25: 1061-1069.

Aráoz S, Joseau MJ, Meehan A \& Hernández R (2016) Calidad física y fisiológica de semillas de Baccharis crispa Sprengel y Baccharis salicifolia Ruiz \& Pav. para su domesticación. Quebracho 24:59-69.

Azcón-Bieto J \& Talón M (2000) Fundamentos de fisiología vegetal. Primera edición interamericana. MG Graw-Hill de España, Madrid. Pp. 169-171.

Balzarini MG, Gonzalez L, Tablada M, Casanoves F, Di Rienzo JA \& Robledo CW (2008) Infostat. Manual del Usuario. Editorial Brujas, Córdoba. 336p.

Baskin JM \& Baskin C (2004) A classification system of seed dormancy. Seed Science Research 14:1-16.

Batlla D \& Benech-Arnold RL (2015) Predicting changes in dormancy level in weed seed soil banks: implications for weed management. Crop Protection 26: 189-197.

Begum MAJ, Selvaraju P \& Venudevan B (2013) Saline stress on seed germination. Scientific 
Research and Essays 8: 1420-1423. Disponible en <https://pdfs.semanticscholar.org/97ab/ d3ccf750b92da9dc94d5d1007dd5adc4fc8b.pdf $>$. Acceso al 28 marzo 2017.

Biganzoli F, Wiegand T \& Batista WB (2009) Firemediated interactions between shrubs in a South American temperate savannah. Oikos 118: 13831395.

Bradford KJ (1995) Water relations in seed germination. In: Kigel J \& Galili G (eds.) Seed development and germination. Marcel Dekker, New York. Pp. 351-396.

Brusa C \& Grela I (2007) Flora arbórea del Uruguay, con énfasis en las especies de Rivera y Tacuarembó. COFUSA, Montevideo. 544p.

Budel JM, Duarte MDR, Santos CDM \& Farago PV (2004) Morfoanatomia foliar e caulinar de Baccharis dracunculifolia DC., Asteraceae. Acta Farmacéutica Bonaerense 23: 477-483.

Cabrera AL (1958) Fitogeografía. In: de Aparicio F \& Difrieri HA (eds.) La Argentina. Suma de geografía. Tomo 3. Peuser, Buenos Aires. Pp. 101-207.

Cabrera AL (1963) Compuestas In: Cabrera AL (ed.) Flora de la Provincia de Buenos Aires. Colección Científica del Instituto Nacional de Tecnología Agropecuaria 4(6a): XIV + 443.

Cabrera AL (1976) Regiones fitogeográficas argentinas. In: Kugler WF (ed.) Enciclopedia argentina de agricultura y jardinería. $2^{\mathrm{a}}$ ed. Tomo 2 . Fascículo 1. Acme, Buenos Aires. Pp. 1-85.

Cantero JJ, Palchetti V, Núñez CO \& Barboza GE (1990) Halophytic flora of Argentina: a checklist and an analysis of its diversity. In: Khan MA, Boër B, Özturk M, Clüsener-Godt M, Gul B \& Breckle S (eds.) Sabkha Ecosystems Volume V: the Americas, tasks for vegetation science 48. Springer International Publishing, Switzerland. Pp: 137-205.

Crisci JV, Freire SE, Sancho G \& Katinas L (2001) Historical biogeography of the Asteraceae from Tandilia and Ventania mountain ranges (Buenos Aires, Argentina). Caldasia 23:21-41.

da Silva Filho AA, Resende DO, Fukui MJ, Santos FF, Pauletti PM, Cunha WR, Silva ML, Gregório LE, Bastos JK \& Nanayakkara NP (2009) In vitro antileishmanial, antiplasmodial and cytotoxic activities of phenolics and triterpenoids from Baccharis dracunculifolia D.C. (Asteraceae). Fitoterapia 80: 478-482.

Di Rienzo JA, Casanoves F, Balzarini MG, Gonzalez L, Tablada M \& Robledo CW (2016) InfoStat Software. Grupo InfoStat, FCA, Universidad Nacional de Córdoba, Córdoba.

Dimitri MJ (1988) Enciclopedia argentina de agricultura y jardinería. $3^{\text {ra }}$ ed. ACME, Buenos Aires. 1160p.

Dürr C, Dickie JB, Yang XY \& Pritchard HW (2015) Ranges of critical temperature and water potential values for the germination of species worldwide: contribution to a seed trait database. Agricultural and Forest Meteorology 200:222-232.

Espírito-Santo MM \& Fernandes GW (1998) Abundance of Neopelma baccharidis (Homoptera: Psyllidae) galls on the dioecious shrub Baccharis dracunculifolia (Asteraceae). Environmental Entomology 27: 870-876.

Espírito-Santo MM, Madeira BG, Neves FS, Faria ML, Fagundes M \& Fernandes GW (2003) Sexual differences in reproductive phenology and their consequences for the demography of Baccharis dracunculifolia (Asteraceae), a dioecious tropical shrub. Annals of Botany 91: 13-19.

Fabiane KC, Ferronatto R, Santos ACD \& Onofre SB (2008) Physicochemical characteristics of the essential oils of Baccharis dracunculifolia and Baccharis uncinella DC (Asteraceae). Revista Brasileira de Farmacognosia 18: 197-203.

Fagundes M \& Fernandes GW (2011) Insect herbivores associated with Baccharis dracunculifolia (Asteraceae): responses of gall-forming and freefeeding insects to latitudinal variation. Revista de Biología Tropical 59: 1419-1432.

Ferracini V, da Silva AG, Paraiba LC, Leitao Filho HF \& Marsaioli AJ (1996) Essential oils of seven Brazilian Baccharis: a prospective approach on their ecological role. In: Caligari PDS \& Hind DJN (eds.) Compositae: biology \& utilization. Proceedings of the International Compositae Conference. Vol. 2. Royal Botanic Gardens, Kew. Pp. 467-474.

Ferreira AG, Cassol B, Rosa SGT, Silveira TS, Stival AL, Silva AA (2001) Germinação de sementes de Asteraceae nativas. Acta Botanica Brasilica 15: 231-242.

Finch-Savage WE \& Leubner-Metzger G (2006) Seed dormancy and the control of germination. New Phytologist 171:501-23.

Frangi JL (1975) Sinopsis de las comunidades vegetales y el medio de las sierras de Tandil (Provincia de Buenos Aires). Boletín de la Sociedad Argentina de Botánica 15: 293-319.

Galíndez G (2008) Arbustización de las sabanas mesopotámicas: Estrategias de regeneración de los arbustos nativos y sus repuestas al fuego y a la herbivoría. Tesis de Doctorado en Agronomía. Universidad de Buenos Aires, Buenos Aires. Pp. 87.

Galíndez G, Ortega-Baes P, Daws MI, Suhring S, Scopel AL \& Pritchard HW (2009) Seed mass and germination in Asteraceae species of Argentina. Seed Science and Technology 37: 786-790.

Galíndez G, Ortega-Baes P, Scopel AL \& Hutchings MJ (2013) The dynamics of three shrub species in a fire-prone temperate savanna: the interplay between the seed bank, seed rain and fire regime. Plant Ecology 214:75-86. 
Giuliano D \& Plos A (2014) Baccharis. Flora Argentina 7: 43-123.

Glenn EP, Brown JJ \& Blumwald E (1999) Salt tolerance and crop potential of halophytes. Critical Reviews in Plant Science 2: 227-255.

Gomes V \& Fernandes GW (2002) Germinaçăo de aquẽnios de Baccharis dracunculifolia D.C. (Asteraceae). Acta Botanica Brasilica 16: 421-427.

Hester MW, Willis LM, Pickens CN \& Dupuis MJ (2012) Enhancement of Barrier Island and Salt Marsh Vegetation Demonstration Project. In: Final report prepared for the office of coastal protection and restoration, Louisiana. Disponible en: $<$ https://www. lacoast.gov/reports/project/TE-53\%20Final\%20 Report.pdf>. Acceso al 28 marzo 2017.

Huo H \& Bradford KJ (2015) Molecular and hormonal regulation of thermoinhibition of seed germination. In: Anderson JV (ed.) Advances in plant dormancy. Springer International Publishing, Cham. Pp. 3-4.

Hurrell J, Bayón N \& Delucchi G (2017) Plantas cultivadas de la Argentina: Asteráceas (=Compuestas). Hemisferio Sur, Buenos Aires. 576p.

International Seed Testing Association - ISTA (2010) Handbook on flower testing. Aster spp 1-5, Ed. ISTA, Zurich, Bassersdorf.

Jankowska-Blaszczuk, M. \& Daws, M. I. 2007. Impact of red: far red ratios on germination of temperate forest herbs in relation to shade tolerance, seed mass and persistence in the soil. Functional Ecology 21: 1055-1062.

Karrfalt RP \& Olson DF Jr. (2008) Asteraceae- Aster family, Baccaris L. In: Bonner FT \& Karrfalt RP (eds.) The woody plant seed manual, a handbook on seeds of trees and shrubs. Part II specific handling methods \& data for 236 genera. USDA FS Agriculture Handbook 727. National Seed Laboratory, Dry Branch. Pp: 291-294. Disponible en <https://www.nsl.fs.fed.us/B\%20genera.pdf $>$. Acceso al 28 marzo 2017.

Koutsovoulou K, Daws MI \& Costas AT (2014) Campanulaceae: a family with small seeds that require light for germination. Annals of Botany 113: 135-143.

Lorenzo D \& Paz D (2004) Capítulo 10: caracterización de fisicoquímica de los aceites esenciales. In: INIA Aromáticas del Uruguay: INIA Estudios en domesticación y cultivo de especies medicinales $\mathrm{y}$ aromáticas nativas, serie FPTA-INIA. Ed. INIA, Canelones. 261 p Disponible en <http:// www2.mgap.gub.uy/BibliotecasdelMGAP/ BibliotecaCentral/Boletines/B8Publicaciones/ arom\%C3\%A1ticas.pdf>. Acceso al 4 abril 2018.

Montalvo AM, Goode LK \& Beyers JL (2010) Plant Profile for Baccharis pilularis. Native plant recommendations for southern California ecoregions. Riverside-Corona Resource Conservation District and U.S. Department of Agriculture, Forest Service,
Pacific Southwest Research Station, Riverside. Disponible en <http://www.rcrcd.com/index. php?option $=$ com_content $\&$ view $=$ article $\&$ id $=88 \&$ Itemid=190>. Acceso al 28 marzo 2017.

Marchesi E (2004) Capítulo 12: especies vegetales autóctonas e introducidas presentes en el territorio de Uruguay consideradas como aromáticas y/o medicinales en relación con los datos de uso popular. L. In: INIA Aromáticas del Uruguay: INIA Estudios en domesticación y cultivo de especies medicinales y aromáticas nativas, serie FPTAINIA. Ed. INIA, Canelones. 261p. Disponible en $<$ http://www2.mgap.gub.uy/BibliotecasdelMGAP/ BibliotecaCentral/Boletines/B8Publicaciones/ arom\%C3\%A1ticas.pdf $>$. Acceso al 4 abril 2018.

Munns RR \& Rawson HM (1999) Effect of salinity on salt accumulation and reproductive development in the apical meristem of wheat and barley. Functional Plant Biology 26: 459-464.

Negreiros D, Esteves D, Fernándes GW, Berbara RLL, Oki Y, Vichiato M \& Chalu C (2014) Growthsurvival tradeoff in the widespread tropical shrub Baccharis dracunculifolia (Asteraceae) in response to a nutrient gradient. Tropical Ecology 55: 167-176.

Park YK, Paredez-Guzman JF, Aguiar CL, Alencar SM, Fujiwara FY (2004) Chemical constituents in Baccharis dracunculifolia as the main botanical origin of southeastern brazilian propolis. Journal of Agricultural and Food Chemestry 52: 1100-1103.

Prado H, van Baren C, Di Leo Lira P, Bandoni A \& Orfila E (2003) El aceite esencial de Baccharis tandilensis Speg. Asteraceae. Dominguezia 19: 20-24.

Requesens E (2011) Bases agroambientales para un desarrollo sustentable del partido de Azul. Facultad de Agronomía de la Universidad Nacional del Centro de la Provincia de Buenos Aires, Azul. 136p.

Sala JM, Kruse E \& Aguglino R (1987) Investigación hidrológica de la Cuenca del Arroyo Azul, Provincia de Buenos Aires. Informe 37. Comisión Investigaciones Científicas de la Provincia de Buenos Aires, La Plata. 235p.

Scaramuzzino RL, Gandini M, Lara B, Bardi JF \& D’Alfonso C (2015) Distribución del arbusto Baccharis dracunculifolia subsp. tandilensis en la provincia de Buenos Aires: cambios en la preferencia de hábitat y su incidencia sobre el paisaje In: Minotti P \& Entraigas I. El paisaje: unidad natural, funcional, dinámica y resiliente. Ed. ASADEP Azul. Pp: 258-260.

Soares Gusman G, Couto Bittencourt AH \& Vestena S (2008) Alelopatia de Baccharis dracunculifolia DC. sobre a germinação e desenvolvimento de espécies cultivadas. Acta Scientiarum Biological Sciences 30: 119-125.

Spegazzini C (1901) Contribución al estudio de la flora del Tandil. Sesé, Larrañaga y Renovales, Buenos Aires. 60p. 
Tardáguila A (2004) Estudios en domesticación y cultivo de especies medicinales y aromáticas nativas. 6. Fichas técnicas de cultivo. Serie 11. Instituto Nacional de Investigación Agropecuaria, Salto. 227p.

Teixeira Duarte MC, Figueira GM, Sartoratto A, Garcia Rehder V L \& Delarmelina C (2005) Anti-Candida activity of Brazilian medicinal plants. Journal of Ethnopharmacology 97: 305-311.

TeKrony DM (2005) Acelerated aging test: principles and procedures. A symposium: seed vigor. Seed Technology 27: 135-145.

Thiers B [continuamente actualizado]. Index Herbariorum: a global directory of public herbaria and associated staff. New York Botanical Garden's Virtual Herbarium. Disponible en $<$ http://sweetgum. nybg. org/ih/>. Acceso en marzo 2017.

Tucat G (2015) Estudios sobre la biología de Baccharis ulicina Hook \& Arn y su susceptibilidad a estrategias de manejo en el sur bonaerense. Tesis de Doctorado. Universidad Nacional del Sur, Bahía Blanca. Disponible en $<$ http://repositoriodigital. uns.edu.ar/handle/123456789/2639>. Acceso a 28 marzo 2017.
Valencia-Díaz S \& Montaña C (2005) Temporal variability in the maternal environment and its effect on seed size and seed quality in Flourensia cernua DC. (Asteraceae). Journal of Arid Environments 63: 686-695. Disponible en <http://www.sciencedirect. com/science/article/pii/S0140196305000820>. Acceso al 28 marzo 2017.

Valicenti RO, Farina E, D'Alfonso C \& Scaramuzzino R (2005). Caracterización fitosociológica de un pajonal serrano de Paspalum quadrifarium Lam. en Azul (provincia de Buenos Aires). Revista Científica Agropecuaria Universidad Nacional de Entre Rios 9: 141-152.

Valicenti R, Farina E, Scaramuzzino R \& D'Alfonso C (2010) Ordenación de la vegetación en el paisaje Boca de la Sierras (Azul, Sistema de Tandilia). Revista de la Sociedad Argentina de Ecología de Paisajes 1: 111-122.

Zuloaga FO, Morrone O \& Rodríguez D (1999) Análisis de la biodiversidad en plantas vasculares de la Argentina. Kurtziana 27: 17-167. 